\title{
CD VIVO: VIABILIZANDO ATIVIDADES DIDÁTICAS COMPUTACIONAIS
}

\author{
Ricardo Andreas Sauerwein, UFSM, rsauer.ufsm@gmail.com \\ Gláucio Carlos Libardoni, UNIJUÍ, glaucio.libardoni@unijui.edu.br \\ Dioni Paulo Pastorio, UFSM, dionipastorio@ hotmail.com \\ Josemar Alves, UFSM, josemarfis@gmail.com
}

\begin{abstract}
Resumo: Neste artigo, entendeu-se que a tecnologia de CD Vivo pode ser usada para viabilizar a implementação de atividades didáticas computacionais (ADC) que usam aplicativos não padronizados. Um CD Vivo é um recurso que pode ser usado para construir ambientes computacionais totalmente voltados para a solução de uma particular ADC. Apresentou-se uma breve discussão sobre CD Vivo e alguns critérios para escolha de versões já existentes que podem ser adaptadas para necessidades específicas. Mostrou-se um CD Vivo que foi criado para atender as necessidades e que foi oferecido como uma das alternativas para realizar ADC. Além disso, exemplificouse sua aplicação com cerca de duzentos alunos, de duas escolas, que resolveram a parte computacional das tarefas propostas, o que permite concluir que o CD Vivo é um recurso didaticamente valioso.
\end{abstract}

Palavras-chave: CD Vivo, atividades didáticas computacionais, ensino de física, software.

\section{LIVE CD: enabling the implementation of computational learning activities}

Abstratct: In this article, it was understood that the live CD technology can be used to enable the implementation of computational learning activities (ADC) that use nonstandard applications. A live CD is a resource that can be used to build computing environments fully focused on the solution of a particular ADC. He presented a brief discussion on live $\mathrm{CD}$ and some criteria for choosing existing versions that can be adapted to specific needs. It proved to be a live CD that was created to meet the needs and what was offered as an alternative to perform ADC. Moreover, exemplified up your application with about two hundred students from two schools, which solved the computational part of the proposed tasks, which shows that the Live CD is a didactically valuable resource.

Keywords: live $\mathrm{CD}$, computational learning activities, physical education, software.

\section{INTRODUÇÃO}

A adoção cada mais vez ampla e frequente do uso de computadores é um fenômeno irreversível (Mota e Scott, 2014). Rapidamente, assim que o computador é adotado em algum ramo da atividade humana, ocorrem grandes e profundas modificações que, praticamente, inviabilizam uma volta a um estágio anterior onde ele não era usado (Lévy, 1998; Coll e Monereo, 2010). Nesse sentido, como o potencial didático do computador é tão vasto e diversificado, pode-se dizer que seu uso e impacto na educação constituem um ramo de pesquisa cada vez mais importante. Isso se traduz no reconhecimento de sua importância didática por diversos autores: (Fiolhais e Trindade, 2003; Giordan, 2005; Heineck, Valiati e Rosa, 2007; Dos Anjos, 2008; Coll e Monereo, 2010; Mota e Scott, 2014). Medeiros e Medeiros (2002) destacam a crescente diversificação e uso das novas tecnologias introduzidas pelos computadores, assim como discutem suas potencialidades e limitações. Fiolhais e Trindade (2003), por sua vez, refletem sobre os possíveis usos do computador como ferramenta didática, como, 
por exemplo, aquisição automática de dados, modelização e simulação, multimídia, realidade virtual e internet.

Paralelamente, iniciativas de políticas públicas, tais como o $\mathrm{UCA}^{1}$ (Um Computador por Aluno) e o Proinfo ${ }^{2}$ (Programa Nacional de Tecnologia Educacional), foram implementadas devido ao reconhecimento da importância do uso do computador na escola, pois a sua adoção é um fator para: (1) manter as escolas atualizadas; (2) viabilizar o desenvolvimento de competências relacionadas com o emprego das TIC; e (3) combater o crescente insucesso escolar. Está claro que iniciativas como essas facilitaram o acesso ao computador a camadas cada vez maiores e diversificadas da sociedade, tornando a sua presença uma realidade no âmbito escolar. Agora, porém, o desafio é fazer um bom uso didático das novas ferramentas que já estão disponíveis (Coll e Monereo, 2010).

Nessa ótica, o computador é uma ferramenta que pode realizar muitas tarefas, sendo sua indústria caracterizada por um elevado grau de dinamismo. Dessa forma, a quantidade de aplicativos computacionais (AC) é imensa e cresce rapidamente. A educação é apenas uma entre muitas áreas que empregam AC. Assim, apenas uma pequena parcela desses aplicativos é criada com propósitos exclusivamente didáticos. Dada a grande quantidade de $\mathrm{AC}$ não didáticos, é provável que o professor perceba alguns desses aplicativos como sendo potencialmente útil para o processo de ensino e aprendizagem dos conteúdos curriculares.

Nesse caso, o professor provavelmente não encontrará dificuldades relacionadas a problemas de disponibilidade e compatibilidade se o aplicativo em questão puder ser considerado padrão ${ }^{3}$. Nesse trabalho, consideramos um aplicativo padrão quando esse domina seu segmento de atuação. Esse domínio é identificado quando outros aplicativos desse segmento: (1) incorporam recursos de exportar e importar documentos eletrônicos nos formatos do aplicativo dominante; e (2) têm interfaces de usuário e funcionalidades muito semelhantes às do aplicativo dominante. Quando o padrão se estabelece, podemos dizer que de certa forma se padroniza também a forma de uso. Logo, do ponto de vista do professor, basta ensinar como se usa um recurso de um aplicativo que segue o padrão (ou aplicativo compatível), que os alunos conseguirão usá-lo nos aplicativos compatíveis de suas preferências.

A situação é bem diferente quando o professor planeja usar um aplicativo que não pode ser considerado um padrão. Pois quando um aplicativo não dominou uma determinada área de atuação, em geral, há mais de um que pode ser utilizado para a realização de uma mesma tarefa. Essas ferramentas podem possuir interfaces de usuário distintas, pois seus comandos e linguagens subjacentes de programação podem ser muito diferentes. Além disso, os documentos gerados por esses aplicativos são, via de regra, incompatíveis (o documento gerado por um não pode ser lido e modificado pelo outro). Portanto, a implementação de atividades didáticas com aplicativos não padronizados pode fazer com que o professor tenha que enfrentar problemas técnicos que as inviabilizem.

Para casos como esses, a tecnologia do CD Vivo ou em inglês Live CD é uma solução que encontramos. Isso porque nele é possível inserir todos os aplicativos necessários à implementação de uma atividade didática em uma única mídia. Além disso, por essa mídia também conter um sistema operacional ${ }^{4}$ (SO) mínimo capaz

\footnotetext{
1 http://www.uca.gov.br/institucional/

2 http://www.fnde.gov.br/programas/programa-nacional-de-tecnologia-educacional-proinfo

${ }^{3}$ São exemplos de aplicativos padrão: Word, Excel, PowerPoint, entre outros.

${ }^{4}$ Neste artigo denominamos SO todo software necessário para implementar a tela na qual o usuário começa a interagir.
} 
inicializar o computador, os problemas de instalação e compatibilidade são resolvidos.

Apesar do nome, o CD Vivo não precisa necessariamente ser um $\mathrm{CD}$, pode ser um pendrive ou qualquer outra mídia removível, ou mesmo uma área da rede. $\mathrm{O}$ importante é que a mídia de armazenamento utilizada esteja na lista de busca das mídias pelas quais o computador busca pelo SO através do qual executa seu processo de inicialização. Chamaremos todos esses casos de CD Vivo, independentemente da mídia de armazenamento utilizada (mesmo um Pendrive Vivo é aqui chamado CD Vivo).

\section{VIVO (LIVE CD)}

Não se deve confundir um $C D$ vivo com um $C D$ interativo. Este último é um recurso didático que se constitui pela tecnologia de hipertexto para criar um conjunto harmônico de textos, imagens, sons, vídeos e simulações que podem ser percorridos pelo estudante, de forma não linear, segundo seus interesses (Piedras et al., 2011).

Diferentemente de um CD interativo que é percebido pelo usuário como um aplicativo sendo executado pelo ambiente computacional no qual normalmente trabalha, o CD Vivo é percebido como sendo um ambiente computacional completamente distinto. Essa característica do CD Vivo é importante, pois pode-se fazer que a tela inicial do computador iniciado pelo CD Vivo esteja completamente adaptada às necessidades didáticas do professor. Idealmente, o CD Vivo deve conter apenas as ferramentas e instruções (eventualmente na forma de hipertexto) necessárias para a realização das tarefas da atividade didática. Uma interface enxuta e direta evita que o aluno perca a concentração, sem saber que ícone ou opção de menu acessar. O CD Vivo não modifica o computador do usuário, nada é instalado no computador hospedeiro. Essa característica também é importante, pois respeita o usuário que quer usar o aplicativo apenas para a realização da tarefa proposta e não quer instalá-lo permanentemente.

Os computadores necessitam de um sistema operacional (SO) para funcionar, sendo este o responsável pela implementação da interface máquina-usuário. A percepção do computador pelo usuário está diretamente ligada ao seu SO. Pode-se dizer que os aplicativos (softwares capazes de realizar tarefas) são instalados sobre o SO. O Windows, Linux, MacOs, entre outros, são exemplos de SO. Quando se liga o computador, este realiza uma pequena série de testes para verificar a integridade da máquina, possíveis dispositivos externos conectados, etc. Em seguida, busca um SO para ser carregado, executa-o até que o processo de inicialização (em inglês boot) esteja completo, quando então, o SO passa a intermediar a comunicação do usuário com os aplicativos. Em geral, o sistema operacional carregado está instalado no disco rígido da máquina. Entretanto é possível fazer a máquina carregar outros sistemas operacionais ${ }^{5}$ que podem estar em um CD ou pendrive. O CD Vivo usa esse fato e oferece um SO que executa completamente a partir do $\mathrm{CD}$ e ignora tudo o que está instalado no computador.

Consideramos que as atividades propostas não devem implicar em custos financeiros para os alunos. Logo, não é o caso de proibir o uso de software proprietário $^{6}$, mas sim, de estimular o uso de software livre, os quais possuem uma característica de construção coletiva (comunidade) e não implica fins lucrativos. Dessa forma, nesse trabalho, vamos considerar apenas soluções que envolvam software livre, a

\footnotetext{
${ }^{5}$ Caso a máquina não busque automaticamente outros SO nas unidades de armazenamento externas (leitores de CD, entrada USB com pendrive conectado, etc.) é preciso configurá-la para fazer isso através da modificação de parâmetros acessados pela BIOS.

${ }^{6}$ Word, Excel, dentre outros.
} 
começar do próprio SO. Por isso, trataremos apenas de CD Vivos baseados no SO Linux.

Há basicamente três maneiras de se obter um CD Vivo do Linux, as quais foram ordenadas em grau crescente de dificuldade e decrescente de flexibilidade ${ }^{7}$, que são: (1) "A partir do zero", consultando a documentação sobre modos e procedimentos de produzi-lo; (2) maneiras semiautomáticas usando programas (scripts) a partir de distribuições Linux normais, como Debian ou Ubuntu, que automatizam mecanismos de geração de um CD Vivo; e (3) modificar ou adaptar um CD Vivo já existente para necessidades desejadas. Todas essas maneiras estão disponibilizadas na rede em diversos sítios que são facilmente encontrados por mecanismos de busca.

A abordagem (3) que requer menos conhecimento computacional é a mais indicada, se já houver disponível um CD Vivo que esteja próximo a um sistema que corresponda às necessidades da atividade que se queira implementar. Em geral, este é o caso, pois há centenas de CD Vivo Linux disponíveis.

Por exemplo, o sítio http://livecdlist.com/ faz uma listagem, não exaustiva, das alternativas existentes. Quando construímos nosso primeiro CD Vivo, em 09/11/2011, esta lista continha 270 variantes, apresentando-as na forma de uma tabela com suas principais características. Através de links na própria tabela, é possível acessar informações mais detalhadas de cada variante de CD Vivo, incluindo a homepage do projeto responsável por sua produção e o endereço em que pode ser baixado. Devido ao grande número de variantes, é muito provável que alguma esteja próxima do que se deseja. Dessa forma, a modificação de uma variante já existente é, via de regra, a mais rápida de ser implementada. Por esse motivo adotamos esse caminho.

Destacamos que ao buscar uma variante de CD Vivo, não consideramos que ele deva conter os aplicativos que queremos usar, porque cada atividade didática pode empregar diferentes aplicativos. Além disso, a maioria dos CD Vivo não tem finalidade didática. Porém, essa finalidade será dada pelas modificações implementadas no mesmo, segundo as necessidades específicas da atividade que se queria realizar. A seguir, discutimos critérios de escolha da variante de CD Vivo que servirá de base para a construção de um CD Vivo didático:

1. Esta variante é disponibilizada pela licença de uso GNU? Este é um requisito essencial. De outro modo não teríamos autorização para adaptar o CD Vivo para nossas necessidades. Uma vez adaptado, o nosso CD Vivo passa ser uma subvariante do original que deve ser distribuído segundo a mesma licença GNU.

2. Esta variante ocupa pouco espaço? O CD mais comum tem uma capacidade de $700 \mathrm{MB}$. Porém é preciso que o SO deixe bastante espaço disponível para aplicativos e materiais de apoio. Além disso, o CD Vivo é executado totalmente na memória RAM, logo, há o risco de variantes muito grandes se tornarem muito "pesadas", ou seja, quanto menor for o SO mais rápido rodará o computador.

3. Esta variante uma vez iniciada apresentará uma interface gráfica amigável para o usuário trabalhar? Este requisito também é fundamental. A maioria dos usuários está acostumada a trabalhar em interfaces que usem o mouse e sejam bastante intuitivas. Aqui há um compromisso com o item anterior, pois quanto mais amigável maior será o tamanho do SO.

4. Esta variante é capaz de iniciar em diferentes tipos de computadores? O ideal é

\footnotetext{
${ }^{7}$ Flexibilidade, para nós, significa controle sobre o produto final. Assim quanto mais controle se deseja sobre o CD Vivo final, mais trabalho se tem em entender as interdependências entre os softwares que são necessárias para se implementar um SO completo e funcional.
} 
que o CD Vivo tenha uma grande compatibilidade de hardware, isto é, execute em máquinas antigas (até 5 anos) e novas.

5. Esta variante é modular e tem grande quantidade de aplicativos já compilados? Requisito essencial. É preciso poder acrescentar e retirar aplicativos facilmente de forma a conseguirmos produzir um CD Vivo que atenda nossas necessidades didáticas.

6. É fácil incorporar material de apoio nesta variante e integrá-lo à interface gráfica do usuário? Uma vez iniciado, o sistema deve apresentar ao usuário um ambiente de trabalho limpo e enxuto no qual se tem rápido acesso a tudo o que é necessário para a realização da tarefa proposta.

7. Esta variante dificulta/proíbe sua instalação no computador hospedeiro? Requisito obrigatório. O sistema iniciado pelo CD Vivo deve ser o mais volátil possível. Uma vez desligada a máquina, essa volta a uma configuração na qual nada foi modificado.

Não fizemos um levantamento comparativo entre as múltiplas variantes de CD Vivo existentes, pois o nosso objetivo era encontrar uma variante que pudesse ser facilmente adaptada às nossas necessidades segundo os critérios acima. Essa busca nos levou a focalizar em apenas duas variantes ${ }^{8}:$ Puppy $^{9}$ e $\operatorname{Slax}^{10}$. Nós as denominaremos respectivamente Linux/Puppy e Linux/Slax para enfatizar que são implementações do sistema operacional Linux. Ambas as versões se mostraram bastante semelhantes quanto aos critérios acima, porém escolhemos o Linux/Slax basicamente pelo, já supracitado, critério 7, pois com essa variante, não há maneira automática de se instalar o Linux/Slax no HD do computador hospedeiro.

Uma vez escolhida a variante Linux/Slax, passamos a adaptá-la a cada necessidade do grupo Métodos e Processos de Ensino e Aprendizagem de Ciência (MPEAC). Dessa forma criamos os seguintes CD Vivo: Linux/Slax/MPEAC/slpa e Linux/Slax/MPEAC/java. O primeiro vem sendo usado para trabalhar atividades didáticas envolvendo o estudo de movimentos. E o segundo para trabalhar com simulações computacionais disponibilizadas em Java, como, por exemplo, as do portal Phe $T^{11}$.

\section{PROBLEMAS PRÁTICOS QUE O CD VIVO RESOLVE}

De forma geral, podemos dizer que o CD Vivo resolve dois tipos de problemas relacionados com as atividades didáticas computacionais (ADC), que são: (1) problemas de compatibilidade do aplicativo; e (2) problemas de uso do aplicativo.

Os primeiros são de certa forma imprevisíveis, já que dependem do computador do aluno, da particular versão do seu sistema operacional, da disponibilidade das bibliotecas utilizadas pelo aplicativo, etc. Isto é, caso alguns alunos tentem instalar o aplicativo recomendado e não consigam executá-lo, toda a atividade didática planejada estará inviabilizada. Isso porque solucionar problemas de instalação de cada aluno é algo que o professor dificilmente tem tempo para fazer.

Caso o aluno instale o aplicativo mas não saiba como utilizá-lo, surgirão problemas técnicos relativos ao seu uso. Porém, destacamos que o desenvolvimento de competências na realização de tarefas associadas ao conteúdo a ser estudado é parte

\footnotetext{
${ }^{8}$ Cabe ressaltar que esta escolha depende - além do peso que cada um atribui à cada um dos critérios acima - da experiência computacional prévia dos usuários, logo, outro grupo, provavelmente escolherá outra distribuição.

${ }^{9}$ www.puppylinux.org.

${ }^{10}$ www.slax.org.

${ }_{11}^{1}$ http://phet.colorado.edu
} 
importante da atividade didática. Logo, criar oportunidades e ensinar o estudante a dominar aplicativos computacionais relevantes é de fundamental importância. Portanto, isso deve constar no planejamento da atividade.

No entanto, quando se usam aplicativos não padronizados, o problema é a multiplicidade de alternativas computacionais que podem realizar a tarefa proposta. $\mathrm{O}$ professor pode não ter condição de ensinar o uso de todas. Uma solução possível é recomendar o uso de um aplicativo, assegurar que o aluno tenha acesso ao mesmo e ensinar as competências necessárias para operá-lo. Estudantes mais independentes no uso do computador, eventualmente podem preferir realizar a tarefa com aplicativos diferentes do recomendado. Naturalmente, a mesma autonomia deve ser mostrada no uso do mesmo, que deve ser feito sem sobrecarregar o professor com dúvidas técnicas de uso de aplicativos não recomendados.

\section{O CD VIVO MPEAC/SLPA}

No primeiro ano do Ensino Médio são geralmente tratados os conteúdos de cinemática e no segundo, o movimento harmônico simples e amortecido. Experimentalmente esses dois temas, quando estudados, geram uma grande quantidade de dados e, consequentemente, para seu entendimento, necessita-se o uso de recursos gráficos.

Normalmente, em livros textos, esses gráficos são mostrados a título de explicação do fenômeno. Porém, temos por pressuposto que a melhor maneira de abordar esse conteúdo é propor tarefas nas quais o próprio aluno constrói e analisa os gráficos, pois julgamos que o conhecimento oriundo da análise e construção é fundamental para o aprendizado dos conteúdos propostos. Assim, esses conteúdos são ideais para serem trabalhados em conjunto com aplicativos computacionais, uma vez que há ferramentas tanto para a aquisição automática dos dados experimentais quanto para sua análise. Nessa seção, mostramos que necessitamos de três aplicativos para trabalhar computacionalmente esse conteúdo.

Os movimentos são descritos através da análise da variação da posição em função do tempo. Hoje em dia, filmadoras estão amplamente disponíveis, pois praticamente qualquer câmera eletrônica, webcam, ou smartphone pode produzir filmes simples. Um arquivo de vídeo contém uma sequência de registros fotográficos tomados a intervalos regulares de tempo. Logo, se o movimento de um objeto for filmado contra um plano de fundo contendo referenciais de posição, o arquivo de vídeo resultante conterá todas as informações necessárias para se fazer um estudo quantitativo do movimento de um objeto.

Nesse sentido, é necessário ainda um aplicativo capaz de ler (abrir) o arquivo de vídeo de forma a poder examinar cada quadro "estático" que o compõe, de forma que se possa extrair informações da posição em função do tempo. Além disso, considerando que os arquivos de vídeo são codificados em vários formatos, definidos pelos fabricantes das câmeras, é importante escolher um aplicativo capaz de ler os formatos mais utilizados. Por esta razão e pela facilidade de uso, escolhemos para esta tarefa o aplicativo Avidemux.

Uma vez tabulados os dados de posição e tempo de cada quadro do vídeo é preciso analisá-los com uma ferramenta adequada. Há várias alternativas possíveis. Escolhemos o Octave pois entendemos que o desenvolvimento de competência em um ambiente de computação numérica $(\mathrm{ACN})$ é algo que os estudantes poderão utilizar por toda sua vida acadêmica e profissional. A escolha do Octave é justificada, visto que, este software é livre e gratuito, o que vai ao encontro da proposta de disseminação de softwares livres discutida anteriormente. Além disso, está disponível na rede, é 
compatível com os principais sistemas operacionais e suas funções condizem com as do software MatLab (proprietário), aplicativo padrão nas áreas de engenharia e afins.

Além disso, o Octave é uma ferramenta que contém uma linguagem de programação subjacente. Os comandos podem ser digitados e interpretados como funções dessa linguagem. Ele pode ser usado interativamente ou então seus comandos podem ser digitados em um editor de texto não formatado (como os editores usados por programadores) de modo que o trabalho possa ser recuperado e estendido.

A realização de atividade didática envolve aplicativos das três áreas: (1) análise de imagem, (2) ACN e (3) edição de texto não formatado. Nós escolhemos para realizar a atividade, respectivamente, os aplicativos Avidemux, Octave e KWrite e criamos um CD Vivo MPEAC/SLPA contendo esses três ${ }^{12}$.

\section{EXEMPLO DE APLICAÇÃO DO CD VIVO}

Desde 2011, temos usado o CD Vivo para implementar atividades didáticas que usam o computador junto a várias iniciativas do grupo Métodos e Processos de Ensino e Aprendizagem em Ciências (MPEAC). Entre as atividades, WorkShop voltado aos professores do Ensino Médio, atividades com bolsistas do PIBID Física/UFSM e atividades direcionadas aos alunos do Ensino Médio em escolas públicas e privadas da cidade de Santa Maria/RS. Também usamos em atividades computacionais para alunos dos primeiros semestres dos cursos de Ciências Exatas da Universidade Federal de Santa Maria (UFSM). Como o uso dessa tecnologia tem se mostrado útil, acreditamos que pode ser usada em outras realidades. Portanto, neste trabalho descrevemos critérios de elaboração de um CD Vivo. Também mostramos como um CD Vivo que criamos foi utilizado nas atividades didáticas computacionais planejadas para trabalhar tópicos do conteúdo da disciplina de Física do nível médio através de atividades computacionais.

Um dos autores do presente trabalho foi professor regente da disciplina de Física em escolas de nível médio na cidade de Santa Maria. Em 2010 atuou em uma escola pública (doravante identificada como escola $\mathrm{A}^{13}$ ) onde lecionou para quatro turmas (100 alunos) do segundo ano do Ensino Médio. Em 2011, transferiu-se para uma escola da rede particular de ensino (doravante identificada como escola $\mathrm{B}^{14}$ ) onde assumiu três turmas (99 alunos) do primeiro ano do Ensino Médio. Ambas as escolas possuíam excelentes estruturas físicas.

Entretanto, apesar de acessarem cotidianamente redes sociais, utilizarem editores de textos eletrônicos e fazerem pesquisas na Internet com grande assiduidade, poucos alunos usavam aplicativos matemáticos ou gráficos na realização de tarefas escolares. Nesse caso, não desenvolviam competências importantes, como análise/construção e interpretação de gráficos, que lhes deveriam acompanhar toda sua vida acadêmica e profissional e que são reconhecidas pelos $\operatorname{PCN}^{15}$ (Brasil, 2002). Sabendo que essas competências só podem ser criadas e mantidas se constantemente usadas, planejamos e implementamos atividades articuladas ao conteúdo programático da disciplina de Física

12 Os detalhes das atividades que desenvolvemos para trabalhar os conteúdos programáticos de Cinemática, Movimento Harmônico Simples e Amortecido com esse CD Vivo fazem parte da dissertação de mestrado: "A inserção de novas tecnologias em conjunto com atividades experimentais no ensino de física" (Libardoni, 2012), disponível em http://www.ufsm.br/ppgecqv.

${ }_{13}$ Através de questionário inicial exposto aos alunos, percebeu-se que nesta escola a maioria dos estudantes tinha acesso a computadores e fazia uso quase diário do mesmo.

${ }^{14}$ Através de questionário inicial exposto aos alunos, percebeu-se que nesta escola a maioria dos estudantes tinha acesso diminuto a computadores e não fazia uso diário do mesmo.

${ }^{15}$ Os PCN são a referência básica para a elaboração das matrizes de referência. Os PCN foram elaborados para difundir os princípios da reforma curricular e orientar os professores na busca de novas abordagens e metodologias. 
e que também desenvolvessem competências na área de análise gráfica e numérica de dados.

Nesse sentido, foram propostas atividades computacionais junto a essas turmas com o objetivo de trabalhar o conteúdo programático da disciplina de Física em conjunto com atividades didáticas computacionais relacionadas ao estudo experimental de movimento. A parte computacional das tarefas propostas poderiam ser realizadas com o conjunto dos três aplicativos reunidos no CD Vivo MPEAC/SLPA apresentado na seção anterior.

Ao propor as atividades esclarecemos que tanto o uso do CD Vivo quanto dos aplicativos sugeridos eram optativos. Também, mostramos aos estudantes que os aplicativos sugeridos, Octave, Avidemux e editores de textos não formatados (equivalentes ao $K W$ rite) estavam disponíveis na Internet, em versões tanto para Linux quanto para Windows, que poderiam ser baixados e instalados diretamente em seus computadores. Contudo, ao mesmo tempo, destacamos que iríamos esclarecer apenas dúvidas relativas ao uso dos aplicativos sugeridos ${ }^{16}$.

As atividades realizadas tanto com os alunos da escola A, quanto da escola B foram planejadas para serem desenvolvidas em grupos de quatro ou cinco membros em horário extraclasse. Inicialmente, distribuímos gratuitamente uma cópia do $\mathrm{CD}$ vivo a cada grupo formado, 20 grupos da escola A e 20 grupos da escola B. Para o início da atividade, em sala de aula, foi mostrado através de exemplo, como configurar a BIOS para iniciar o computador via CD Vivo. Além disso, também os computadores dos laboratórios de informática das respectivas escolas foram ajustados para iniciar automaticamente pelo CD vivo, a fim de otimizar o tempo de realização da atividade.

Com o objetivo de validar a iniciativa do uso CD vivo, classificamos os modos pelos quais os grupos de alunos resolveram as atividades propostas segundo os critérios:

(1) O CD Vivo foi acessado no computador de um dos integrantes do grupo;

(2) O CD Vivo foi acessado no computador de mais de um integrante do grupo;

(3) O grupo baixou da Internet os aplicativos sugeridos;

(4) O grupo optou por usar outros aplicativos, como, por exemplo, o Origin;

(5) O grupo optou por não utilizar o computador.

$\mathrm{Na}$ tabela 1, as duas últimas colunas indicam, respectivamente, o número de grupos das Escolas A e B que acessaram o CD Vivo, segundo os modos descritos acima e que estão indicados na primeira coluna.

Tabela 1 - Número de Grupos x Acesso ao CD Vivo.

\begin{tabular}{|c|c|c|}
\hline Modo & Grupos da Escola A & Grupos da Escola B \\
\hline 1 & 5 & 8 \\
\hline 2 & 9 & 10 \\
\hline 3 & 4 & 0 \\
\hline 4 & 2 & 2 \\
\hline 5 & 0 & 0 \\
\hline
\end{tabular}

${ }^{16}$ Como mencionado anteriormente, é inviável o professor sanar dúvidas de todos os vários aplicativos que poderiam ser utilizados. 
Os grupos que utilizaram os $\mathrm{CD}$ segundo os modos 1 e 2 são aqueles que reconheceram o CD Vivo como uma facilidade proporcionada para a realização da tarefa computacional proposta. A tabela 1 mostra que a grande maioria dos grupos, $80 \%$ (32/40), optou por utilizar o CD Vivo, logo mostra a importância da iniciativa do CD Vivo como ferramenta computacional.

Os grupos que utilizaram o CD Vivo segundo os modos 3 e 4 eram compostos de alunos com habilidades no uso de ferramentas computacionais e optaram por não utilizar o CD Vivo. A tabela 1 mostra que apenas 15\% (6/40) dispensaram o uso do CD Vivo. Esses grupos são todos da Escola A, que de maneira geral, possuem mais vivência no uso de computadores. Ainda segundo o quadro 1, vemos que 5\% (2/40) dos grupos preferiram fazer a tarefa sem o uso do computador. Os dois grupos são da Escola B, que têm menos experiência em tarefas computacionais. Podemos dizer que uma minoria de $10 \%(2 / 20)$ dos grupos da escola B eram formados por alunos que evitavam o uso de computador e sempre que possível usavam a estratégia de calculadora, lápis e papel.

A partir da proposta, podemos conjecturar o que teria ocorrido se o CD Vivo não estivesse disponível. Uma das consequências possíveis, seria que o tempo de implementação teria sido maior. Além disso, certamente alguns alunos menos familiarizados com o computador não conseguiriam instalar os aplicativos necessários para a execução das tarefas. Por exemplo, o Octave e o Avidemux são softwares livres que são facilmente instalados no Linux. Porém, a maioria dos estudantes usa o Windows, onde a instalação do software livre costuma ser mais complexa e muitos estudantes não conseguem fazê-la. Tanto o aumento do tempo gasto na realização da atividade quanto problemas técnicos relacionados à instalação dos aplicativos requeridos podem comprometer a realização de atividades computacionais que usam aplicativos não padronizados. Logo, a observação das maneiras como os estudantes das escolas A e B realizaram as tarefas computacionais propostas mostra que o CD Vivo é um recurso valioso que viabilizou atividades didáticas.

\section{CONSIDERAÇÕES FINAIS}

No grupo MPEAC o uso de CD Vivo tem se mostrado bastante útil em espaços de aprendizagem formal, pois é uma maneira de solucionar problemas que são comumente encontrados em atividades computacionais que usam aplicativos não padronizados. O CD Vivo certamente resolve o problema de compatibilidade do aplicativo, pois, caso o aluno não consiga ou não queira instalar um aplicativo em seu computador, ele pode usar a alternativa proporcionada pelo CD Vivo. Quanto aos problemas relacionados às dificuldades de uso dos aplicativos, podemos dizer que o CD Vivo oferece uma plataforma comum, na qual as instruções de uso do aplicativo podem ser dadas. Assim, ao oferecer o CD Vivo como alternativa para a realização de uma ADC também será oferecido aos alunos um caminho viável para realizá-las.

Nesse trabalho, mostramos um caso em que $80 \%$ dos grupos de alunos preferiram usar o CD Vivo para a realização de tarefas computacionais, mesmo esse sendo um recurso optativo. No entanto, temos observado que esse recurso pode viabilizar ADC em outras circunstâncias. Por exemplo, já nos deparamos com laboratórios de informática de escolas que proíbem a instalação de aplicativos. O CD Vivo é claramente uma ferramenta que contorna essa situação, porque mantém os computadores hospedeiros intactos. Outra situação em que o CD Vivo tem se mostrado bastante útil se dá em oficinas voltadas aos professores. Eles podem conhecer e experimentar novas ferramentas computacionais sem o compromisso de instalá-las em seus computadores. Essa é uma maneira de vencer a resistência que muitas pessoas ainda têm em relação ao uso de software livre ou do sistema operacional Linux. 


\section{REFERÊNCIAS}

BRASIL. Ministério da Educação. Secretaria da Educação Média e Tecnológica. Parâmetros Curriculares Nacionais+ (PCN+) - Ciências da Natureza e suas Tecnologias. Brasília: MEC, 2002.

COLL, C.; MONEREO, C. Psicologia da Educação Virtual: Aprender e ensinas com as tecnologias da informação e da comunicação. Tradução Naila Freitas. Porto Alegre: Artmed, 2010. 365 p.

DOS ANJOS, A. J. S. As novas tecnologias e o uso dos recursos telemáticos na educação científica: a simulação computacional na educação em Física. Caderno Brasileiro de Ensino de Física, v. 25, n. 3, p. 569-600, 2008.

FIOLHAIS, C.; TRINDADE, J. Física no Computador: O Computador como uma Ferramenta no Ensino e na Aprendizagem das Ciências Físicas. Revista Brasileira de Ensino de Física, v. 25, n.3, p.259-272, 2003.

GIORDAN, M. O computador na educação em ciências: breve revisão crítica acerca de algumas formas de utilização. Ciência \& Educação, v. 11, n. 2, p. 279-304, 2005.

HEINECK, R.; VALIATI, E. R. A.; ROSA, C. T. W. Software educativo no ensino de Física: análise quantitativa e qualitativa. Revista Iberoamericana de Educación, v. 42, n. 6, p. 1-12, 2007.

LÉVY, P. A revolução contemporânea em matéria de comunicação. Tradução Juremir Machado da Silva. Revista FAMECOS: mídia, cultura e tecnologia, v. 1, n. 9, p. 3749, 1998.

LIBARDONI, G. C. A inserção de novas tecnologias em conjunto com atividades experimentais no ensino de física. Santa Maria: UFSM, 2012. 108p. Dissertação de Mestrado.

MEDEIROS, A.; MEDEIROS, C. F. Possibilidades de limitações das simulações computacionais no ensino da física. Revista Brasileira de Ensino de Física, v. 24, n 2, p. 77-86, 2002.

MOTA, R.; SCOTT, D. Educando para inovação e aprendizagem independente. Rio de Janeiro: Elsevier, 2014.

PIEDRAS, E. M. R.; ARAUJO, A. P.; BORDA, A; A. S.; HERRMANN, A. C.; ORTIZ, J. O. Representação gráfica digital para o design através de ambientes virtuais de aprendizagem. Novas Tecnologias na Educação, v. 9, n. 2, p. 1-10, 2011. 